\title{
VARIABILIDADE ESPACIAL DE ATRIBUTOS QUÍMICOS E PRODUTIVIDADE DA CULTURA DO CAFÉ EM DUAS SAFRAS AGRÍCOLAS
}

\author{
Spatial variability of chemical attributes and coffee productivity in two harvests \\ Fábio Moreira da Silva ${ }^{1}$, Zigomar Menezes de Souza², Carlos Augusto Pereira de Figueiredo ${ }^{3}$, \\ Luciano Henrique de Souza Vieira ${ }^{3}$, Ezequiel de Oliveira $^{4}$
}

\begin{abstract}
RESUMO
O emprego da Agricultura de Precisão pode ser uma das alternativas para o país continuar com a liderança mundial de café, por meio da competitividade nos custos e na qualidade do produto. Objetiva-se com este trabalho, avaliar a variabilidade espacial dos atributos químicos do solo e da produtividade da cultura do cafeeiro em duas safras agrícolas. Amostras de solo foram coletadas na profundidade de $0,0-0,2 \mathrm{~m}$, nos pontos de cruzamento de uma malha com intervalos regulares de $25 \mathrm{~m}$, perfazendo um total de 68 pontos em uma área de 6,2 ha. Os frutos de quatro plantas, em torno dos pontos de cruzamento da malha, foram coletados para o cálculo da produtividade média por planta. A dependência espacial encontrada para os atributos químicos e produtividade da cultura do café, bem como a semelhança de comportamento dos atributos estudados nas diferentes safras, permite inferir que o efeito de bianuidade não interferiu na variabilidade espacial da cultura do café. A grande amplitude nos atributos químicos justifica o estudo da aplicação diferenciada e localizada de fertilizantes na lavoura cafeeira. A regressão linear espacial mostrou comportamento semelhante da variabilidade espacial dos atributos químicos e produtividade da cultura do café nas duas safras estudadas.
\end{abstract}

Termos para indexação: Agricultura de precisão, krigagem, latossolo, Coffea arabica.

\begin{abstract}
Precision agriculture is one of the alternatives that may help maintaining the Brazilian international leadership in coffee production by reducing production costs and increasing competitiveness and product quality. Assessing the spatial variability of soil attributes has a crucial importance for the application of precision agriculture techniques. Therefore, the aim of this work was to evaluate the spatial variability of soil chemical attributes and coffee yield in two agricultural seasons. Soil samples were collected in a depth of 0.0-0.2 m, at the crossing points of a regular grid, comprising a total of 68 points located at $25 \mathrm{~m}$-intervals in an area of 6.2 ha. Fruits of four coffee plants around the soil sampling points were collected and the average yield per plant was calculated. The spatial dependence found for chemical attributes and coffee yield as well as the similar behavior of the attributes studied in the different agricultural seasons indicates that the bi-annuity effect did not intervene with the spatial variability of the crop. The large amplitude found for soil chemical attributes justifies to study the differentiated and localized application of fertilizers in coffee plantations. The spatial linear regression showed a similar spatial variability in both chemical attributes and coffee yield in the two studied seasons.
\end{abstract}

Index terms: Site-specific management, kriging, oxisol, Coffea arabica.

\section{(Recebido em 22 de setembro de 2006 e aprovado em 7 de fevereiro de 2007)}

\section{INTRODUÇÃo}

Com a globalização da economia e a competitividade de preço dos produtos agrícolas, surgiu a necessidade de se obter níveis de competitividade internacionais. Além disto, a busca pela conservação dos recursos naturais, impõe à atividade agrícola novos métodos e técnicas de produção, aliados à eficiência e maior controle dos resultados obtidos no campo, em relação ao que se pratica hoje. Sendo que a Agricultura de Precisão (AP), apresenta um conjunto de tecnologia capaz de auxiliar o produtor rural a identificar as estratégias a serem adotadas para aumentar a eficiência no gerenciamento da agricultura, maximizando a rentabilidade das colheitas e tornando o agronegócio mais competitivo.

Para as culturas de cereais, os métodos e equipamentos necessários para adoção de AP já são relativamente bem conhecidos, e fazem parte, inclusive, de equipamentos já comercializados no país. Esse não é o

\footnotetext{
1Doutor, Professor Adjunto - Departamento de Engenharia/DEG - Universidade Federal de Lavras/UFLA - Cx. P. 3037 - $37200-000$ - Lavras, MG fmsilva@ufla.br

${ }^{2}$ Doutor, Professor Faculdade de Engenharia Agrícola/FEAGRI - Universidade Estadual de Campinas/UNICAMP - Cx. P. 6011 - 13.083-875 Campinas, SP - zigomarms@yahoo.com.br

${ }^{3}$ Graduandos - Departamento de Engenharia/DEG - Universidade Federal de Lavras/UFLA - Cx. P. 3037 - $37200-000$ - Lavras, MG gutoengenharia@hotmail.com

"Mestre - Departamento de Engenharia/DEG - Universidade Federal de Lavras/UFLA - Cx. P. 3037 - 37200-000 - Lavras, MG - Lavras, MG ezequielufla@yahoo.com.br
} 
caso de culturas especiais como a de laranja, de cana-deaçúcar e de café, onde não existem, ou são poucos, os trabalhos publicados sobre a utilização dos conceitos de AP (BALASTREIRE et al., 2001).

Vários autores ressaltam a importância em conhecer a variabilidade dos atributos nas unidades de mapeamento do solo, principalmente para a interpretação de levantamentos técnicos, como aptidão agrícola e capacidade de uso, para estimar a densidade de amostragem ideal em levantamentos de solos (BERG \& KLAMT, 1997), e atualmente para aplicação do conceito de manejo localizado, ou seja, AP (DODERMANN \& PING, 2004).

As relações entre variabilidade espacial dos atributos do solo e a variabilidade dos atributos de plantas, têm sido estudadas para ajudar a identificar a relação de causa e efeito desses atributos (MILLER et al., 1988; SALVIANO et al., 1998). A dependência espacial de atributos do solo e da planta, pode ser descrita utilizandose o semivariograma experimental (ISAAKS \& SRIVASTAVA, 1989), ao qual se pode ajustar um modelo matemático, conforme descrito por Mcbratney \& Webster (1986). Esse modelo é usado no processo de interpolação, denominado krigagem, para estimar valores em locais não amostrados.

Sanchez et al. (2005), estudando a variabilidade espacial de atributos químicos do solo e da produção de café em diferentes superfícies geomórficas, observaram dependência espacial para todos os atributos químicos estudados e para produtividade do café. Molin et al. (2002) realizaram um trabalho de mapeamento de produtividade de café e sua correlação com o componente de fertilidade do solo, em duas áreas pilotos nos municípios de Gália-SP e Pompéia-SP. Observaram que os componentes de correlação entre a produtividade e a fertilidade do solo resultaram em baixos valores. As duas áreas apresentaram grande variação na produtividade de 22 a $75 \mathrm{sc} \mathrm{ha}^{-1}$.

No caso da cultura do cafeeiro, há um vasto campo para pesquisa em que a AP possa ser aplicada, pela importância da cultura para nosso país, pelo alto grau tecnológico que tem-se observado em muitas lavouras e pelo seu valor econômico. No entanto, o número de pesquisas com AP e a caracterização da variabilidade espacial com a cultura do café é pequena. Portanto, conduziu-se este trabalho para avaliar a variabilidade espacial de atributos químicos e a produtividade da cultura do cafeeiro, em duas safras agrícolas.

\section{MATERIAL E MÉTODOS}

O experimento foi conduzido na fazenda Cafua, localizada no município de Ijací, sul de Minas Gerais, em uma área de 6,2 ha de lavoura da cultivar Mundo Novo, com doze anos de idade, plantada no espaçamento de $4 \mathrm{~m}$ entre linhas e $1 \mathrm{~m}$ entre plantas, totalizando 2.500 plantas ha-1. As coordenadas geográficas são de $21^{\circ} 10^{\prime} 11^{\prime \prime}$ de latitude sul e $44^{\circ} 58^{\prime} 37^{\prime \prime}$ de longitude oeste, com altitude média de 934 metros e uma declividade de $0,84 \%$, no sentido longitudinal, e $12 \%$ no sentido transversal. O clima da região foi classificado pelo método de Köppen, como subtropical, com inverno seco (Cwb).

Nas safras de 2002-2003 e 2003-2004 foram coletados dados da produtividade de quatro plantas em torno dos pontos amostrais durante o mês de julho, sendo considerada a produtividade em coco média por planta (L planta ${ }^{-1}$ ). Para cada ponto da grade amostral (pontos georeferenciados) foram coletadas na linha de plantio, com auxílio de um trado, amostras compostas (4 sub-amostras) de solo, na profundidade de $0,0-0,2 \mathrm{~m}$. O solo foi amostrado nos pontos de cruzamento de uma malha, com intervalos regulares de $25 \mathrm{~m}$, perfazendo um total de 68 pontos, na profundidade de $0,0-0,2 \mathrm{~m}$. O solo foi classificado como Latossolo Vermelho-Amarelo distrófico. A área vem recebendo uma adubação anual de $180 \mathrm{~g} \mathrm{cova}^{-1}$ da fórmula 26-0-26 e 2,5 $\mathrm{kg} \mathrm{ha}^{-1}$ de zinco e boro, em adubação foliar.

$\mathrm{Na}$ caracterização química do solo foi determinada potenciometricamente, a acidez ativa ( $\mathrm{pH}$ em água). As bases trocáveis cálcio $(\mathrm{Ca})$ e magnésio $(\mathrm{Mg})$ foram extraídas utilizando o extrator $\mathrm{KCl} 1 \mathrm{~N}$ e o fósforo disponível (P), e, para o potássio $(\mathrm{K})$ o extrator foi o mehlich-1 (EMBRAPA, 1997). Com base nos resultados das análises químicas, foi calculada a soma de base (SB) e capacidade de troca catiônica (CTC).

Os dados foram, inicialmente, avaliados por meio da estatística descritiva, tomando por base as seguintes medidas: média, mediana, variância, coeficiente de variação, coeficientes de assimetria e de curtose. A hipótese de normalidade dos dados foi verificada pelo teste de Kolmogorov-Smirnov, por meio do programa computacional SAS (SCHLOTZHAVER \& LITTELL, 1997). A estatística clássica foi utilizada para avaliar a variabilidade dos dados e se o conjunto de dados tem distribuição normal. Quando os valores da média, mediana e moda apresentam valores semelhantes, os dados apresentam ou aproximam da distribuição normal (LITTLE \& HILLS, 1978). Em conjunto com essas estatísticas, temse que, uma distribuição normal apresenta coeficiente de assimetria e curtose próximo a zero, embora alguns programas estatísticos utilizem uma maneira de cálculo do coeficiente de curtose que resulta em três.

A geoestatística, ferramenta estatística utilizada para estudar a variabilidade espacial, possibilita a interpretação 
dos resultados com base na estrutura da variabilidade natural dos atributos avaliados, considerando a dependência espacial dentro do intervalo de amostragem. A dependência espacial foi avaliada com base nas pressuposições de estacionaridade da hipótese intrínseca (VIEIRA, 2000), pela análise de semivariogramas ajustados pelo software $\mathrm{GS}^{+}$- GAMMA DESIGN SOFTWARE (ROBERTSON, 1998). O semivariograma foi estimado pela seguinte expressão:

$$
\hat{\gamma}(\mathrm{h})=\frac{1}{2 \mathrm{~N}(\mathrm{~h})} \sum_{\mathrm{i}=1}^{\mathrm{N}(\mathrm{h})}\left[\mathrm{Z}\left(\mathrm{x}_{\mathrm{i}}\right)-\mathrm{Z}\left(\mathrm{x}_{\mathrm{i}}+\mathrm{h}\right)\right]^{2}
$$

onde $\mathrm{N}$ (h) é o número de pares experimentais de observações, $\mathrm{Z}\left(\mathrm{x}_{\mathrm{i}}\right)$ e $\mathrm{Z}\left(\mathrm{x}_{\mathrm{i}}+\mathrm{h}\right)$, separados por uma distância h. O semivariograma estimado é representado pelo gráfico $\hat{\gamma}(h)$ versus h. Do ajuste de um modelo matemático aos valores calculados de $\hat{\gamma}(h)$, são estimados os coeficientes do modelo teórico para o semivariograma (o efeito pepita, $\mathrm{C}_{0}$; patamar, $\mathrm{C}_{0}+\mathrm{C}_{1}$; e o alcance, a).

Os modelos de semivariogramas testados foram o esférico, o exponencial, o linear e o gaussiano (McBRATNEY \& WEBSTER, 1986), sendo ajustados pelo programa computacional $\mathrm{GS}^{+}$. No caso de mais de um modelo adequado para o mesmo semivariograma, foi escolhido o de maior valor do coeficiente de correlação, obtido pelo método de validação cruzada e o de melhor coeficiente de determinação $\left(\mathrm{R}^{2}\right)$. Para elaboração dos mapas de distribuição espacial das variáveis foi utilizado o programa SURFER (GOLDEN SOFTWARE INC, 1999).

Com o uso da krigagem, foram gerados mapas de isolinhas (contorno), para cada atributo do solo e produtividade da cultura do café. Posteriormente, os mapas gerados no programa SURFER foram exportados para o programa Idrisi, tornando possível a realização da regressão linear simples espacial entre mapas das variáveis estudadas, pixel-a-pixel. Para análise dos resultados referentes à correlação linear espacial foi utilizado o critério empregado por Jakob et al. (1999), verificando o valor da estatística F (Fisher) e levando-se em conta o valor do $\mathrm{R}^{2}$.

Para analisar o grau da dependência espacial dos atributos em estudo, utilizou-se a classificação de Cambardella et al. (1994), em que são considerados de dependência espacial forte os semivariogramas que têm um efeito pepita menor ou igual a $25 \%$ do patamar, moderada quando está entre 25 e $75 \%$ e fraca quando for maior que $75 \%$.

\section{RESULTADOS E DISCUSSÃO}

Os resultados referentes à análise descritiva para os atributos químicos e produtividade da cultura do café foram observados na Tabela 1. Quanto aos resultados referentes ao teste Kolmogorov-Smirnov, verifica-se normalidade para o Mg na safra agrícola de 2002-2003 e o Ca, SB e CTC na safra agrícola de 2003-2004. Os valores da média e mediana, para todas as variáveis, estão próximos, mostrando distribuições simétricas, o que pode ser confirmado pelos valores de assimetria próximos de zero, com exceção da variável fósforo e a produtividade na safra de 2003-2004. De acordo com Isaaks \& Srivastava (1989) o coeficiente de assimetria é mais sensível a valores extremos do que a média, mediana e o desvio padrão, uma vez que um único valor pode influenciar fortemente o coeficiente de assimetria, pois os desvios entre cada valor e a média são elevados à terceira potência.

É importante que nos semivariogramas evidenciemse patamares bem definidos e que na distribuição não se verifique caudas muito alongadas, o que poderia comprometer as estimativas da krigagem, as quais são baseadas nos valores médios (ISAAKS \& SRIVASTAVA, 1989). Corá et al. (2004), estudando a variabilidade espacial de atributos químicos do solo para a cultura de cana-deaçúcar não encontraram normalidade para as variáveis em estudo.

Coeficientes de variação (CV) são adimensionais e observa-se a comparação de valores entre diferentes atributos do solo. Valores elevados de CV considera-se como os primeiros indicadores da existência de heterogeneidade nos dados (FROGBROOK et al., 2002). A variabilidade de um atributo pode ser classificada, segundo Warrick \& Nielsen (1980), de acordo com a magnitude do seu coeficiente de variação. A variabilidade do $\mathrm{pH}$ foi baixa, nas safras agrícolas de 2002-2003 e 2003-2004. Já para os atributos Ca, K, SB e CTC a variabilidade foi média e os atributos $\mathrm{Mg}, \mathrm{P}$ e produtividade apresentaram $\mathrm{CV}$ alto, variando entre $31,15 \%$ e 78,57 \%, nas safras agrícolas estudadas.

$\mathrm{O}$ alto valor de $\mathrm{CV}$ para o fósforo, é provavelmente devido aos efeitos residuais de adubações anteriores, haja vista que este elemento apresenta baixa mobilidade e geralmente é adicionado na linha de plantio, enquanto a correção da acidez do solo, bem como a deposição dos restos culturais, é realizada a lanço, de forma homogênea sobre a área. Segundo Silva et al. (2003) mesmo as aplicações a lanço, seguidas de revolvimento do solo para incorporação do calcário geram variabilidade no solo. 
Tabela 1 - Estatística descritiva para as variáveis $\mathrm{pH}$, cálcio $\left(\mathrm{cmol}_{\mathrm{c}} \mathrm{dm}^{-3}\right)$, magnésio $\left(\mathrm{cmol}_{\mathrm{c}} \mathrm{dm}^{-3}\right)$, potássio $\left(\mathrm{mg} \mathrm{dm}^{-3}\right)$, fósforo $\left(\mathrm{mg} \mathrm{dm}^{-3}\right)$, soma de base $\left(\mathrm{cmol}_{\mathrm{c}} \mathrm{dm}^{-3}\right)$, capacidade de troca catiônica $\left(\mathrm{cmol}_{\mathrm{c}} \mathrm{dm}^{-3}\right)$ de amostras coletadas na malha na profundidade de 0,0-0,2 $\mathrm{m}$ e produtividade da cultura do cafeeiro (L planta $\left.{ }^{-1}\right)$.

\begin{tabular}{|c|c|c|c|c|c|c|c|c|}
\hline Estatística & $\mathrm{pH}$ & $\mathrm{Ca}$ & $\mathrm{Mg}$ & $\mathrm{K}$ & $\mathrm{P}$ & SB & CTC & Produtividade \\
\hline \multicolumn{9}{|c|}{ Safra Agrícola (2002-2003) } \\
\hline Média & 6,74 & 4,96 & 1,46 & 205,91 & 5,58 & 6,47 & 8,01 & 5,91 \\
\hline Mediana & 7,10 & 4,50 & 1,50 & 204,75 & 4,05 & 6,52 & 8,07 & 4,95 \\
\hline Variância & 0,24 & 1,01 & 0,23 & 2127,33 & 5,83 & 1,34 & 0,66 & 6,87 \\
\hline Assimetria & $-0,95$ & 0,32 & $-0,21$ & 0,12 & 0,44 & $-0,33$ & 0,04 & 0,29 \\
\hline Curtose & 0,93 & 0,82 & $-0,39$ & 0,80 & $-0,69$ & $-0,09$ & $-0,28$ & $-0,43$ \\
\hline${ }^{1} \mathrm{CV}$ & 6,89 & 22,53 & 32,77 & 22,39 & 52,72 & 17,89 & 10,12 & 53,36 \\
\hline${ }^{2} \mathrm{~d}$ & 0,13 & 0,15 & $0,07^{\mathrm{ns}}$ & 0,12 & 0,14 & 0,13 & 0,15 & 0,13 \\
\hline \multicolumn{9}{|c|}{ Safra Agrícola (2003-2004) } \\
\hline Média & 6,62 & 3,99 & 1,28 & 186,10 & 4,18 & 5,75 & 7,47 & 4,81 \\
\hline Mediana & 6,70 & 4,01 & 1,30 & 187,12 & 3,96 & 5,80 & 7,50 & 4,75 \\
\hline Variância & 0,22 & 0,87 & 0,07 & 2906 & 9,30 & 1,16 & 0,52 & 18,20 \\
\hline Assimetria & 0,58 & 0,59 & 0,34 & 0,14 & 2,02 & 0,07 & 0,42 & 2,44 \\
\hline Curtose & $-0,55$ & 1,17 & $-0,09$ & $-0,86$ & 5,42 & 0,14 & 0,22 & 7,41 \\
\hline $\mathrm{CV}$ & 7,13 & 23,12 & 31,15 & 21,12 & 72,83 & 18,73 & 12,67 & 78,57 \\
\hline d & 0,19 & $0,10^{\mathrm{ns}}$ & 0,12 & 0,12 & 0,20 & $0,09^{\mathrm{ns}}$ & $0,08^{\mathrm{ns}}$ & 0,21 \\
\hline
\end{tabular}

${ }^{1} \mathrm{CV}=$ coeficiente de variação; ${ }^{2} \mathrm{~d}=$ teste de normalidade, ${ }^{\text {ns }}$ não significativo pelo teste de Kolmogorov-Smirnov.

Pelos resultados da análise geoestatística, concluiu-se que, todas as variáveis analisadas apresentaram dependência espacial (Tabela 2 e Figuras 1 e 2). Os atributos estudados apresentaram coeficientes de determinação do teste de validação cruzada acima de 0,70 , indicando que os modelos ajustados aos semivariogramas dos atributos químicos e produtividade do café estão coerentes. Nas safras agrícolas de 2002-2003 e 2003-2004, o modelo esférico ajustou-se aos dados de todas as variáveis em estudo. Segundo Grego \& Vieira (2005) o modelo matemático esférico é o que predomina nos trabalhos em ciência do solo. McBratney \& Webster (1986) estudaram modelos de ajuste do semivariograma para os atributos do solo e relataram que os modelos esférico e exponencial são os mais encontrados.

$\mathrm{Na}$ análise do grau de dependência espacial das variáveis em estudo, utilizou-se a classificação de Cambardella et al. (1994). A análise da relação $C_{0} /\left(C_{0}+C_{1}\right)$ de todas as variáveis em estudo mostrou grau de dependência espacial forte, com exceção da variável $\mathrm{Mg}$ que apresentou grau de dependência espacial moderado na safra agrícola de 2002-2003, já na safra agrícola de 2003-
2004 as variáveis $\mathrm{pH}, \mathrm{Ca}, \mathrm{Mg}$ e produtividade apresentaram grau de dependência espacial forte e as demais, grau moderado de dependência espacial (Tabela 2).

Para os atributos químicos, evidenciou-se valor de coeficiente de variação elevado (Tabela 1), e, de modo geral, também, o valor de efeito pepita elevado (Tabela $1 \mathrm{e}$ Figuras 1 e 2). Segundo Siqueira (2006) é possível concluirse, por meio da estatística descritiva atributos que apresentaram valores de variabilidade não detectada pelo esquema de amostragem, mostrando-se maior descontinuidade entre amostras.

A distribuição dos atributos químicos e a produtividade da cultura do café, no espaço, não são aleatórias, uma vez que todos apresentaram valores moderados ou fortes para o grau de dependência espacial, conforme estabelecido pela relação $\mathrm{C}_{0} /\left(\mathrm{C}_{0}+\mathrm{C}_{1}\right)$. Verificou-se que $88 \%$ e $90 \%$ da variação total da produtividade de grãos foi explicada pela dependência espacial deste atributo, ao qual, por outro lado, foi atribuído o erro devido ao acaso, de $12 \%$ e $10 \%$, referente ao efeito pepita, nas safras agrícolas de 20022003 e 2003-2004, respectivamente. 
Tabela 2 - Modelos e parâmetros estimados dos semivariogramas experimentais para as variáveis $\mathrm{pH}$, cálcio $\left(\mathrm{cmol}_{\mathrm{c}} \mathrm{dm}^{-3}\right)$, magnésio $\left(\mathrm{cmol}_{\mathrm{c}} \mathrm{dm}^{-3}\right)$, potássio $\left(\mathrm{mg} \mathrm{dm}^{-3}\right)$, fósforo $\left(\mathrm{mg} \mathrm{dm}^{-3}\right)$, soma de bases $\left(\mathrm{cmol}_{\mathrm{c}} \mathrm{dm}^{-3}\right)$, capacidade de troca catiônica $\left(\mathrm{cmol}_{\mathrm{c}} \mathrm{dm}^{-3}\right)$ de amostras coletadas na malha na profundidade de $0,0-0,2 \mathrm{~m}$ e produtividade da cultura do cafeeiro $(\mathrm{L}$ planta $\left.^{-1}\right)$.

\begin{tabular}{|c|c|c|c|c|c|c|c|c|}
\hline Parâmetro & $\mathrm{pH}$ & $\mathrm{Ca}$ & $\mathrm{Mg}$ & K & $\mathrm{P}$ & SB & CTC & Produtividade \\
\hline \multicolumn{9}{|c|}{ Safra Agrícola (2002-2003) } \\
\hline Modelo & ${ }^{1}$ Esf & Esf & Esf & Esf & Esf & Esf & Esf & Esf \\
\hline Efeito pepita $\left(\mathrm{C}_{0}\right)$ & 0,03 & 0,09 & 0,06 & 579 & 0,09 & 0,17 & 0,09 & 0,58 \\
\hline Patamar $\left(\mathrm{C}_{0}+\mathrm{C}_{1}\right)$ & 0,19 & 0,71 & 0,19 & 3622 & 3,45 & 1,08 & 0,48 & 5,02 \\
\hline Alcance (a) & 51,12 & 54,02 & 98,00 & 142,08 & 86,05 & 72,11 & 60,08 & 65,04 \\
\hline$\left[\mathrm{C}_{0} /\left(\mathrm{C}_{0}+\mathrm{C}_{1}\right)\right] \times 100$ & 16 & 13 & 32 & 16 & 3 & 16 & 19 & 12 \\
\hline${ }^{2} \mathrm{VC}$ & 0,72 & 0,70 & 0,72 & 0,75 & 0,70 & 0,70 & 0,73 & 0,72 \\
\hline${ }^{3} \mathrm{R}^{2}$ & 66 & 69 & 95 & 84 & 88 & 72 & 80 & 70 \\
\hline \multicolumn{9}{|c|}{ Safra Agrícola (2003-2004) } \\
\hline Modelo & Esf & Esf & Esf & Esf & Esf & Esf & Esf & Esf \\
\hline Efeito pepita $\left(\mathrm{C}_{0}\right)$ & 0,01 & 0,17 & 0,007 & 973 & 0,52 & 0,27 & 0,16 & 0,79 \\
\hline Patamar $\left(\mathrm{C}_{0}+\mathrm{C}_{1}\right)$ & 0,12 & 0,71 & 0,03 & 2328 & 1,05 & 0,99 & 0,41 & 8,09 \\
\hline Alcance (a) & 72,91 & 67,42 & 65,35 & 188,02 & 210,04 & 62,41 & 98,45 & 60,43 \\
\hline$\left[\mathrm{C}_{0} /\left(\mathrm{C}_{0}+\mathrm{C}_{1}\right)\right] \times 100$ & 8 & 23 & 23 & 42 & 50 & 27 & 39 & 10 \\
\hline $\mathrm{VC}$ & 0,85 & 0,88 & 0,89 & 0,91 & 0,90 & 0,90 & 0,89 & 0,88 \\
\hline $\mathrm{R}^{2}$ & 67 & 91 & 68 & 93 & 84 & 82 & 81 & 61 \\
\hline
\end{tabular}

${ }^{1}$ Esf $=$ esférico $;{ }^{2} \mathrm{VC}=$ coeficiente de determinação do teste de validação cruzada; ${ }^{3} \mathrm{R}^{2}=$ coeficiente de determinação.

Com relação ao alcance da dependência espacial (Tabela 2), os atributos químicos em estudo apresentaram valores bastante próximos nas safras agrícolas de 20022003 e 2003-2004. Com exceção da variável P, para a qual evidenciou-se um valor de alcance bem inferior na safra agrícola de 2002-2003. De maneira geral, os atributos químicos em estudo e a produtividade podem ser amostrados em um espaçamento aproximado de $90 \mathrm{~m}$, com exceção do K nas safras agrícolas em estudo e o P na safra agrícola de 2003-2004, pois apresentaram valores de alcance bem maiores que os demais atributos químicos avaliados. Todas as variáveis em estudo nos levaram a valores baixos de alcance, com exceção das variáveis $\mathrm{P}$ e K, evidenciando a baixa continuidade estrutural do solo sob cafeeiro.

Os mapas das variáveis $\mathrm{pH}, \mathrm{Ca}, \mathrm{Mg}, \mathrm{K}, \mathrm{P}, \mathrm{SB}, \mathrm{CTC}$ e produtividade da cultura do cafeeiro apresentaram, nas safras agrícolas de 2002-2003 e 2003-2004, uma grande amplitude nos atributos químicos estudados (Figuras 3 e 4). Resultados semelhantes foram obtidos por Sanchez et al. (2005), estudando a variabilidade espacial de atributos químicos e produtividade do café e Corá et al. (2004) para cultura da cana-de-açúcar. A grande amplitude revela os problemas que podem ocorrer quando se usa a média dos valores para o manejo da fertilidade, pois em alguns locais da área a aplicação de fertilizante será inferior à dosagem necessária e em outras haverá aplicação excessiva.

Observa-se que os valores médios dos atributos químicos e produtividade da cultura do café foi maior na safra agrícola de 2002-2003 em relação à safra agrícola de 2003-2004 (Tabela 1), o que também pode ser observado pelos mapas de krigagem (Figuras 3 e 4). A baixa produtividade na safra de 2002-2003, deve-se ao efeito de bianuidade da cultura do café. A bianuidade é uma característica fisiológica do cafeeiro que, um ano produz muitos frutos e no outro, independente dos tratos culturais, produz pouco.

No programa Idrisi foi realizada a análise de regressão linear simples espacial entre mapas das variáveis estudadas, pixel-a-pixel $(0,2 \times 0,2 \mathrm{~m})$. A correlação apresentada entre os mapas de $\mathrm{pH}, \mathrm{Ca}, \mathrm{Mg}, \mathrm{K}, \mathrm{P}, \mathrm{SB}, \mathrm{CTC}$ e produção, nas safras agrícolas de 2002-2003 e 2003-2004, foram de $63 \%, 67 \%, 58 \%, 75 \%, 61 \%, 65 \%, 55 \%$ e $69 \%$, respectivamente. A dependência espacial encontrada para os atributos químicos e produtividade da cultura do café, bem como a semelhança de comportamento dos atributos estudados nas diferentes safras (correlação espacial), permite inferir que o efeito de bianuidade não interferiu na variabilidade espacial da cultura do café. 


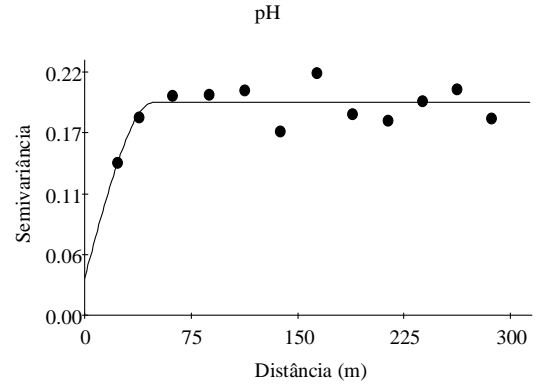

Esf $(0,03-0,19-51)$

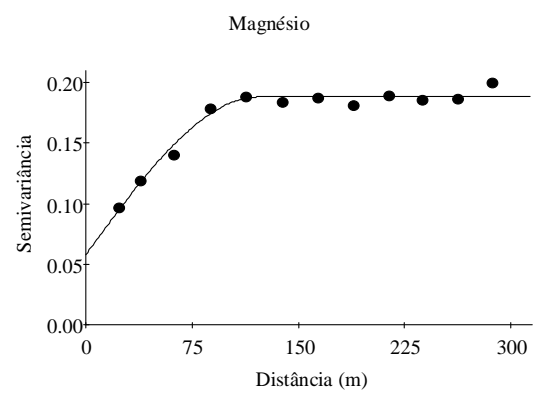

Esf (0,06-0,19-98)

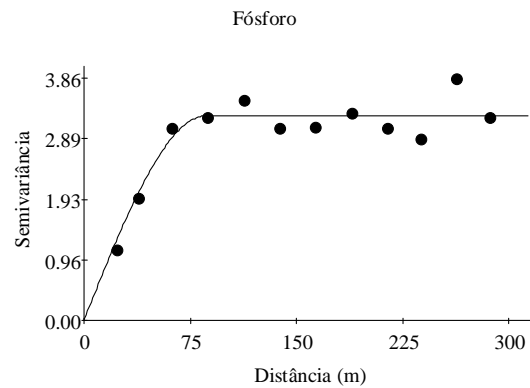

Esf $(0,09-3,45-86)$

Capacidade de Troca Catiônica

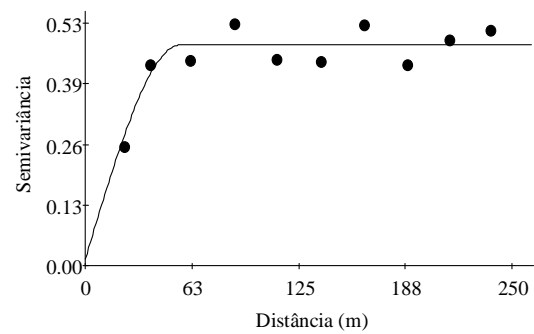

Esf $(0,09-0,48-60)$

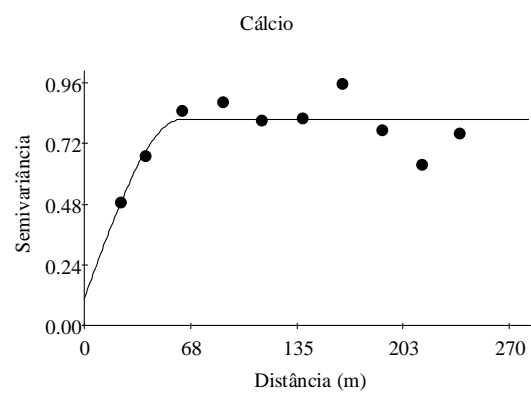

Esf (0,09-0,71-54)

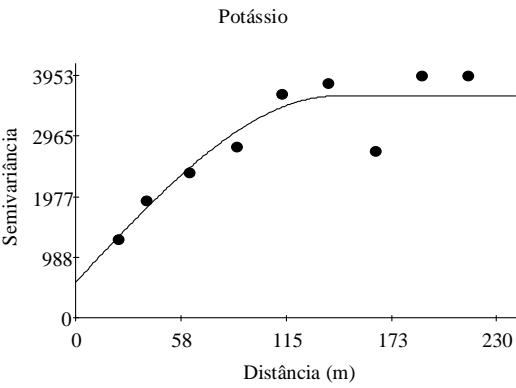

Esf (579-3622-142)

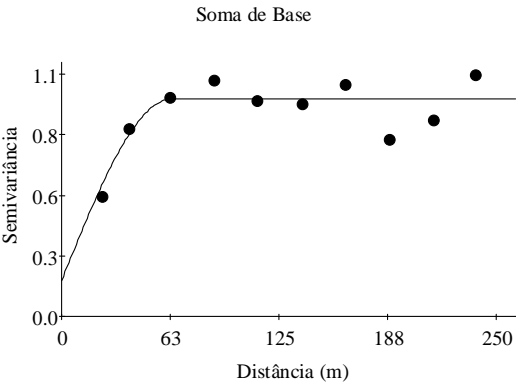

Esf $(0,17-1,08-72)$

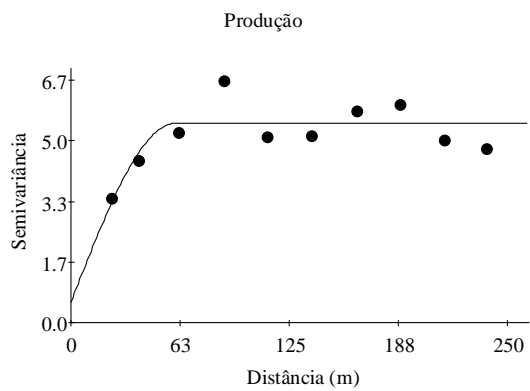

Esf $(0,58-5,02-65)$

Figura 1 - Semivariogramas das variáveis químicas: cálcio $\left(\mathrm{cmol}_{c} \mathrm{dm}^{-3}\right)$, magnésio $\left(\mathrm{cmol}_{c} \mathrm{dm}^{-3}\right)$, potássio $\left(\mathrm{mg} \mathrm{dm}^{-3}\right)$, fósforo $\left(\mathrm{mg} \mathrm{dm}^{-3}\right)$, soma de base $\left(\mathrm{cmol}_{\mathrm{c}} \mathrm{dm}^{-3}\right)$, capacidade de troca catiônica $\left(\mathrm{cmol}_{\mathrm{c}} \mathrm{dm}^{-3}\right)$ e $\mathrm{pH}$ na profundidade de $0,0-$ $0,2 \mathrm{~m}$ e produção da cultura do café $\left(\mathrm{L}_{\text {planta }}{ }^{-1}\right)$, na safra agrícola de 2002-2003. Esf $\left(\mathrm{C}_{\mathrm{o}} ; \mathrm{C}_{0}+\mathrm{C}_{1} ;\right.$ a) é o modelo esférico ajustado, $\mathrm{C}_{\mathrm{o}}=$ efeito pepita; $\mathrm{C}_{0}+\mathrm{C}_{1}=$ patamar; $\mathrm{a}=$ alcance. 


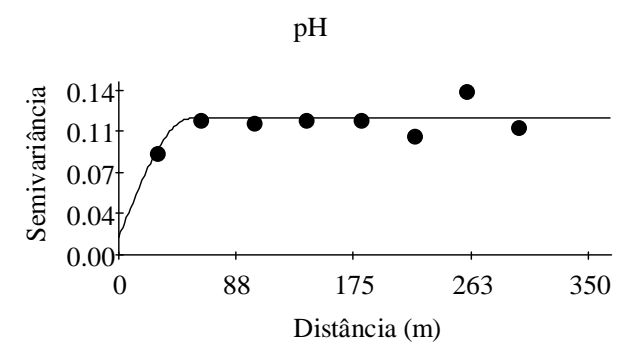

Esf $(0,01-0,12-72,9)$

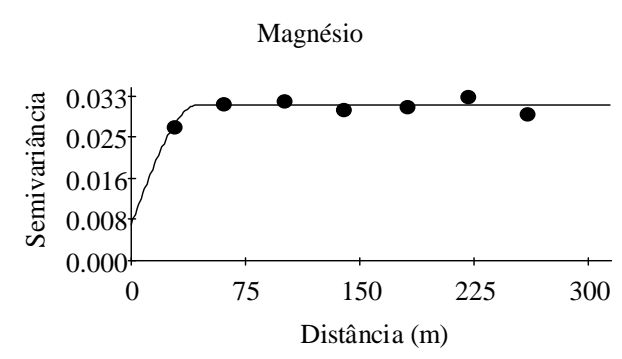

Esf $(0,007-0,03-65,3)$

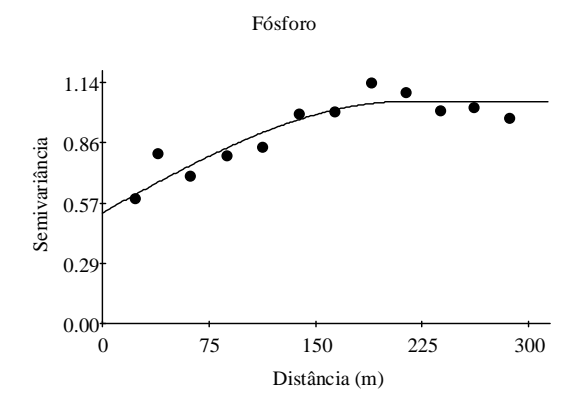

Esf $(0,52-1,05-210)$

Capacidade de Troca Catiônica

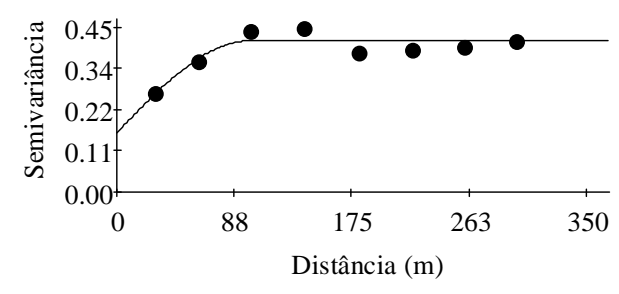

Esf $(0,16-0,41-98,4)$

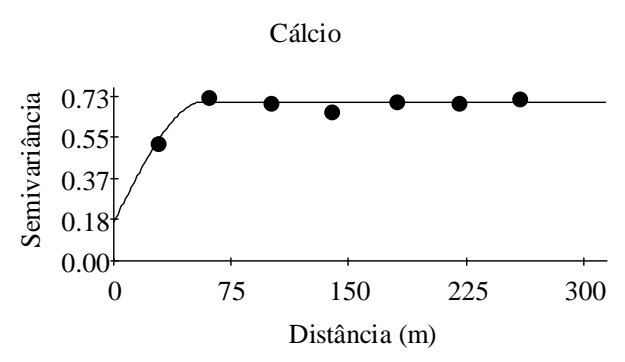

Esf $(0,17-0,71-67,4)$

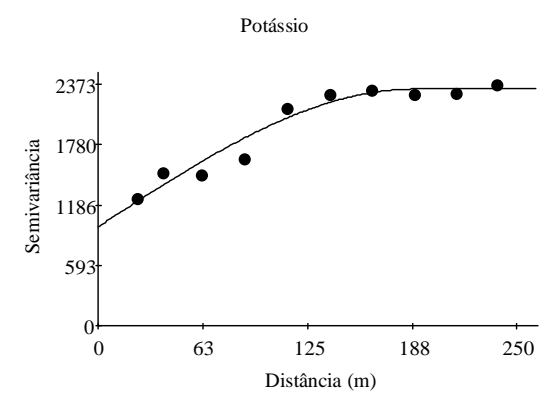

Esf (973-2328-188)

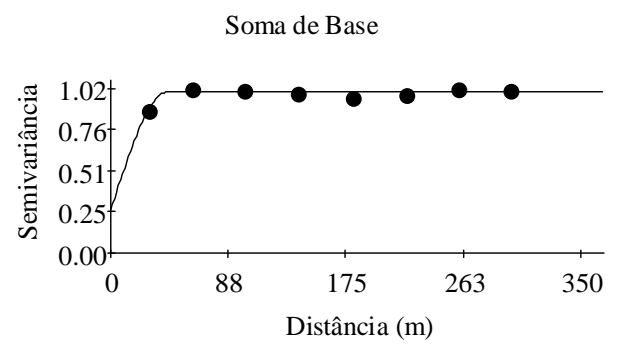

Esf $(0,27-0,99-62,4)$

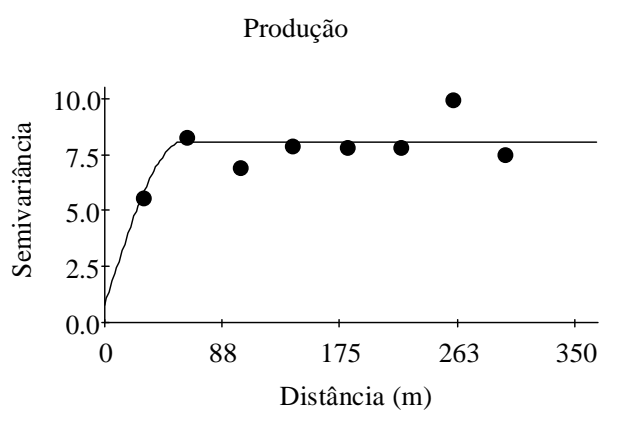

Esf $(0,79-8,09-60,4)$

Figura 2 - Semivariogramas das variáveis químicas: cálcio $\left(\mathrm{cmol}_{\mathrm{c}} \mathrm{dm}^{-3}\right)$, magnésio $\left(\mathrm{cmol}_{\mathrm{c}} \mathrm{dm}^{-3}\right)$, potássio $\left(\mathrm{mg} \mathrm{dm}^{-3}\right)$, fósforo $\left(\mathrm{mg} \mathrm{dm}^{-3}\right)$, soma de base $\left(\mathrm{cmol} \mathrm{dm}_{\mathrm{c}}^{-3}\right)$, capacidade de troca catiônica $\left(\mathrm{cmol}_{\mathrm{c}} \mathrm{dm}^{-3}\right), \mathrm{e} \mathrm{pH}$ na profundidade de $0,0-$ $0,2 \mathrm{~m}$ e produção da cultura do café $\left(\mathrm{L}_{\text {planta }}{ }^{-1}\right)$, na safra agrícola de 2003-2004. Esf $\left(\mathrm{C}_{\mathrm{o}} ; \mathrm{C}_{0}+\mathrm{C}_{1} ; \mathrm{a}\right)$ é o modelo esférico ajustado, $\mathrm{C}_{\mathrm{o}}=$ efeito pepita; $\mathrm{C}_{0}+\mathrm{C}_{1}=$ patamar; $\mathrm{a}=$ alcance. 

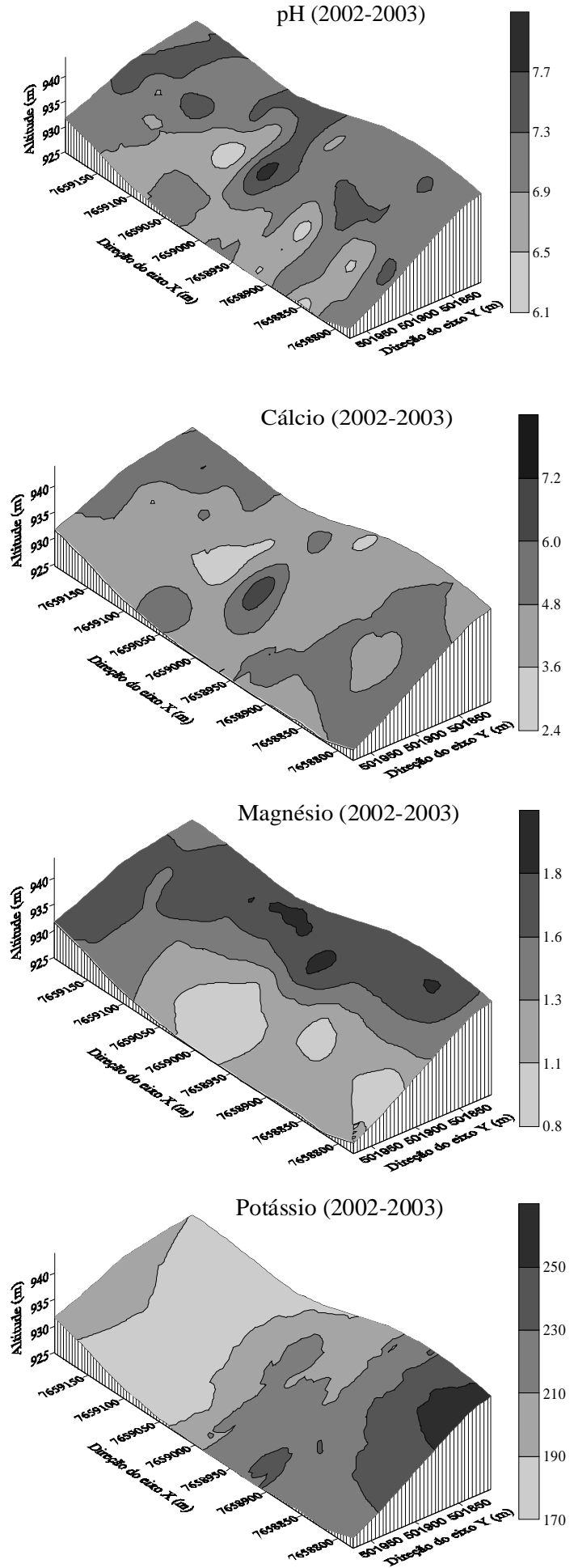
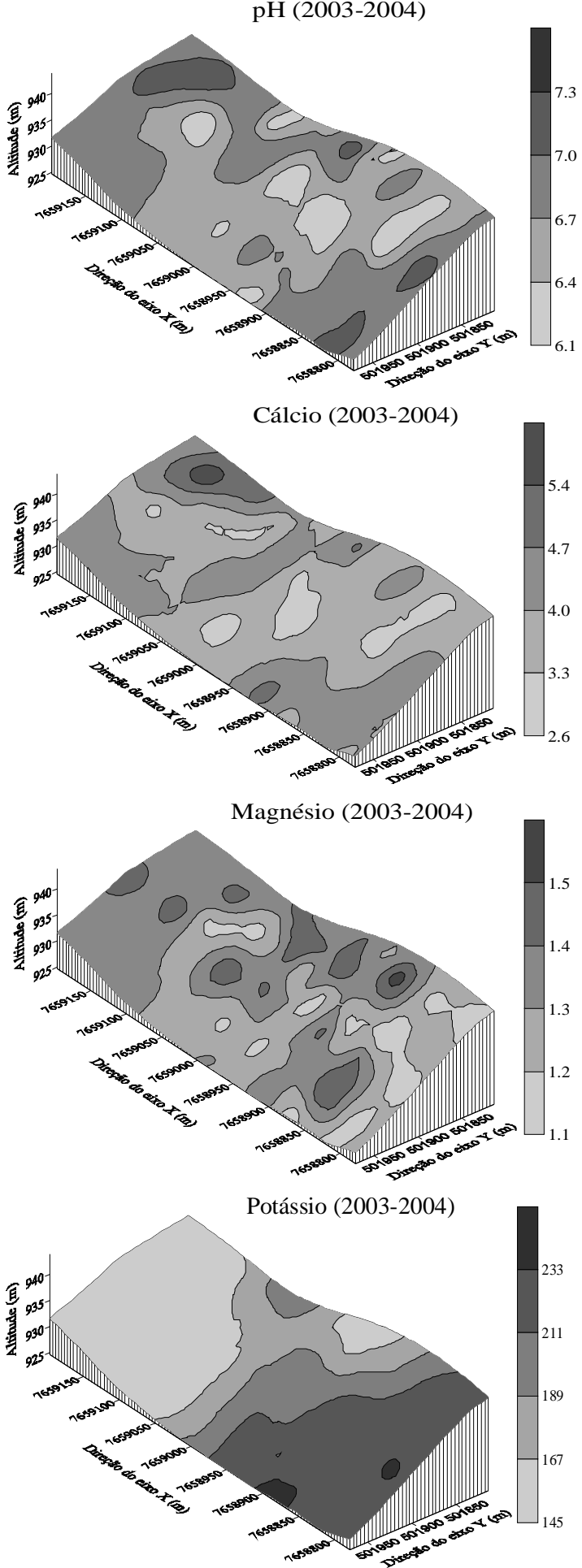

Figura 3 - Distribuição espacial do $\mathrm{pH}$, cálcio $\left(\mathrm{cmol}_{\mathrm{c}} \mathrm{dm}^{-3}\right)$, magnésio $\left(\mathrm{cmol}_{\mathrm{c}} \mathrm{dm}^{-3}\right)$ e potássio $\left(\mathrm{mg} \mathrm{dm}^{-3}\right)$ na profundidade de 0,0-0,2 m nas safras agrícolas de 2002-2003 e 2003-2004. 

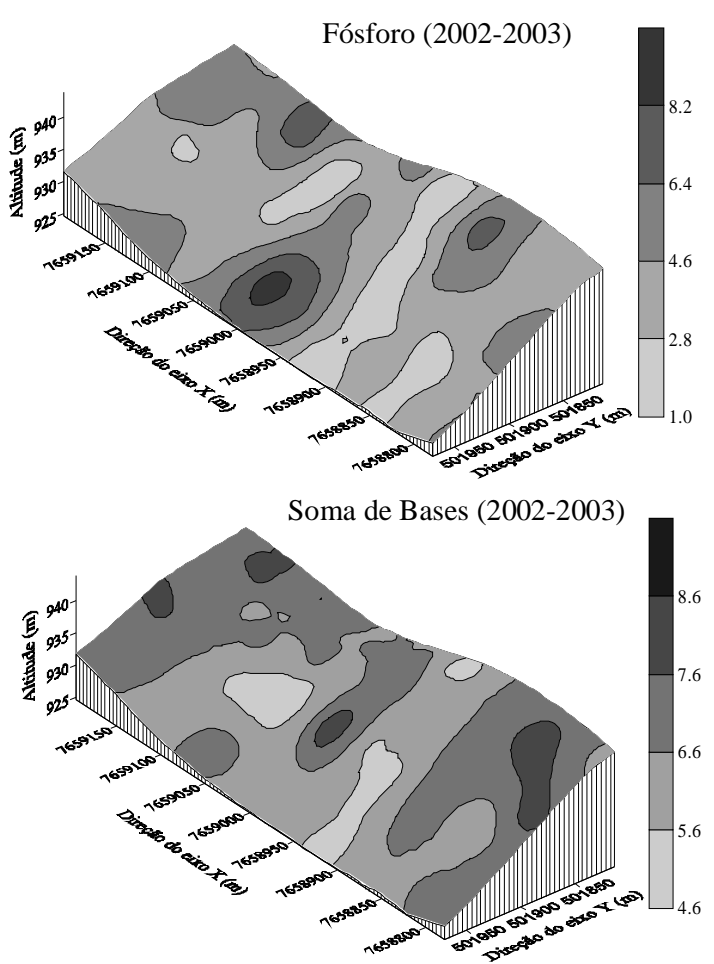

Capacidade de Troca Catiônica (2002-2003)
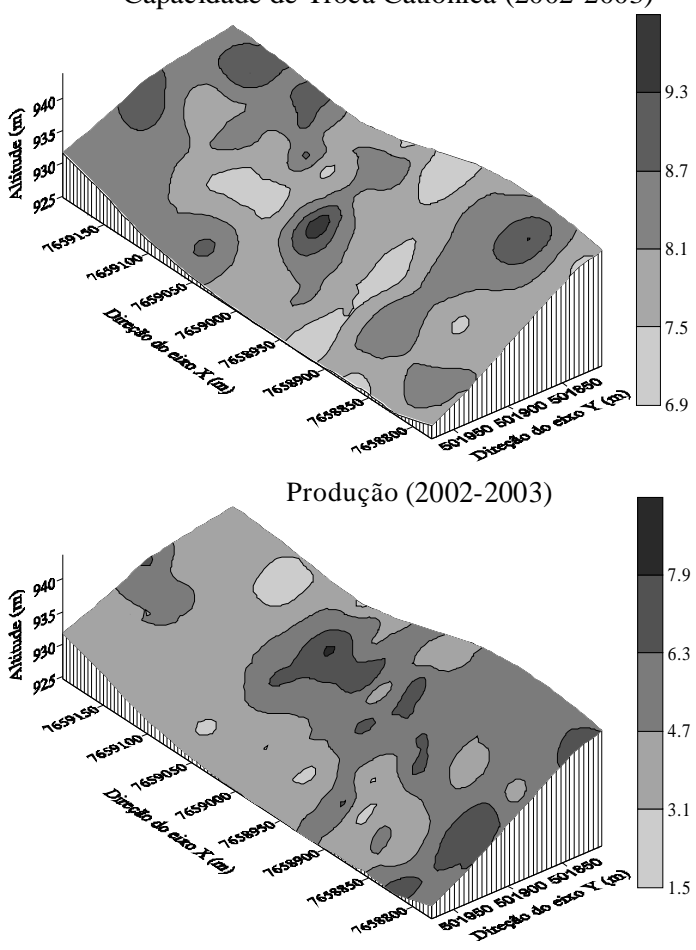
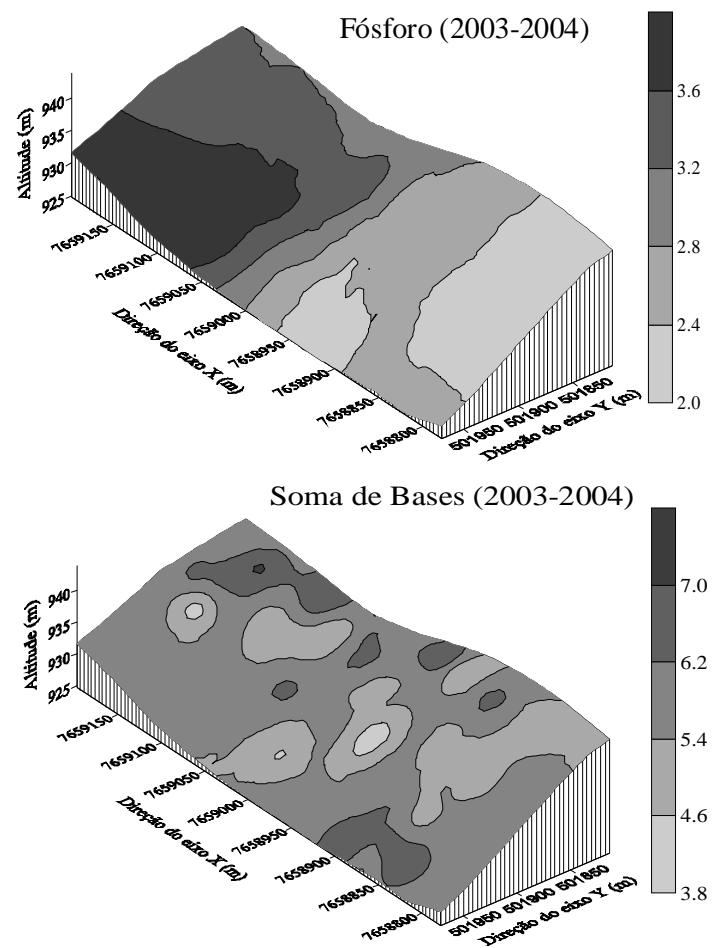

Capacidade de Troca Catiônica (2003-2004)
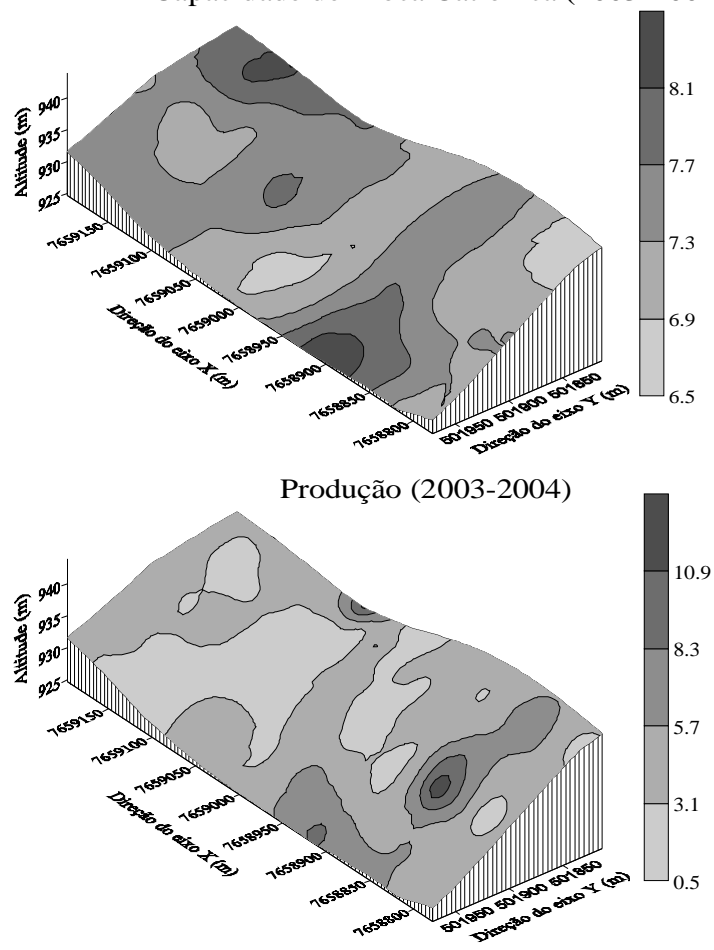

Figura 4 - Distribuição espacial do fósforo $\left(\mathrm{mg} \mathrm{dm}^{-3}\right)$, soma de bases $\left(\mathrm{cmol}_{\mathrm{c}} \mathrm{dm}^{-3}\right)$, capacidade de troca catiônica $\left(\mathrm{cmol}_{\mathrm{c}}\right.$ $\mathrm{dm}^{-3}$ ), na profundidade de 0,0-0,2 m e produtividade da cultura do cafeeiro (L planta ${ }^{-1}$ ), nas safras agrícolas de 2002-2003 e 2003-2004. 


\section{CONCLUSÕES}

A dependência espacial, encontrada para os atributos químicos e produtividade da cultura do café, bem como a semelhança de comportamento dos atributos estudados nas diferentes safras, permite inferir que o efeito de bianuidade não interferiu na variabilidade espacial da cultura do café.

A grande amplitude nos atributos químicos justifica o estudo da aplicação diferenciada e localizada de fertilizantes na lavoura cafeeira.

Para a regressão linear espacial evidenciou-se comportamento semelhante na variabilidade espacial dos atributos químicos e a produtividade da cultura do café, nas duas safras estudadas.

\section{REFERÊNCIAS BIBLIOGRÁFICAS}

BALASTREIRE, L. A.; AMARAL, J. R.; LEAL, J. C. G.; BAIO, F. H. R. Agricultura de precisão: mapeamento da produtividade de uma cultura de café (compact disc). In: CONGRESSO BRASILEIRO DE ENGENHARIA AGRÍCOLA, 30., 2001, Foz do Iguaçu. Anais... Jaboticabal: SBEA, 2001.

BERG, M. V. D.; KLAMT, E. Variabilidade especial de características de solos na região do Planalto Médio, RS: II. análise da semivariância e da variância. Revista Brasileira de Ciência do Solo, Viçosa, v. 21, n. 3, p. 401408, 1997.

CAMBARDELLA, C. A.; MOORMAN, T. B.; NOVAK, J. M.; PARKIN, T. B.; KARLEN, D. L.; TURCO, R. F.; KONOPKA, A. E. Field scale variability of soil properties in Central Iowa soils. Soil Science Society of America Journal, Madison, v. 58, n. 5, p. 1501-1511, 1994.

CORÁ, J. E.; ARAUJO, A. V.; PEREIRA, G. T.; BERALDO, J. M. G. Variabilidade espacial de atributos do solo para adoção do sistema de agricultura de precisão na cultura de cana-de-açúcar. Revista Brasileira de Ciência do Solo, Viçosa, v. 28, n. 6, p. 1013-1021, 2004.

DODERMANN, A.; PING, J. L. Geostatistical integration of yield monitor data and remote sensing improves yield maps. Agronomy Journal, Madison, v. 96, n. 1, p. 285-297, 2004.

EMPRESA BRASILEIRA DE PESQUISA AGROPECUÁRIA. Manual de métodos de análise de solo.
2. ed. Rio de Janeiro: Ministério da Agricultura e do Abastecimento, 1997. 212 p.

FROGBROOK, Z. L.; OLIVER, M. A.; SALAHI, M.; ELLIS, R. $\mathrm{H}$. Exploring the spatial relations between cereal yield and soil chemical properties and the implications for sampling. Soil Use and Management, Avenue, v. 18, n. 1, p. 1-9, 2002.

GOLDEN SOFTWARE INC. Surfer for windows: realese 7.0: contouring and 3D surface mapping for scientist's engineers user's guide. New York, 1999.619 p.

GREGO, C. R.; VIEIRA, S. R. Variabilidade espacial de propriedades físicas do solo em uma parcela experimental. Revista Brasileira de Ciência do Solo, Viçosa, v. 29, n. 2, p. 169-177, 2005.

ISAAKS, E. H.; SRIVASTAVA, R. M. An introduction to applied geoestatistics. New York: Oxford University, 1989. $561 \mathrm{p}$.

JAKOB, A. A. E.; ROCHA, J. V.; LAMPARELLI, R. A. C. Estudo da correlação entre mapas de variabilidade de propriedades do solo e mapas de produtividade para fins de agricultura de precisão (compact disc). In: CONGRESSO E FEIRA PARA USUÁRIOS DE GEOPROCESSAMENTO DAAMÉRICALATINA-GIS BRASIL 99, 5., 1999, Salvador. Anais... Salvador: UFBA, 1999.

LITTLE, T. M.; HILLS, F. J. Agricultural experimentation. New York: J. Wiley \& Sons, 1978. 350 p.

McBRATNEY, A. B.; WEBSTER, R. Choosing functions for semi-variograms of soil properties and fitting them to sampling estimates. Journal Soil Science, Oxford, v. 37, n. 3, p. 617-639, 1986.

MILLER, M. P.; SINGER, M. J.; NIELSEN, D. R. Spatial variability of wheat yield and soil properties on complex hills. Soil Science Society of America Journal, Madison, v. 52, n. 4, p. 1133-1141, 1988.

MOLIN, J. P.; RIBEIRO FILHO, A. C.; TORRES, F. P.; SHIRAISI, L. E.; SARTORI, S.; SARRIÉS, G. A. Mapeamento da produtividade de café e sua correlação com componentes de fertilidade do solo em duas áreas pilotos. In: BALASTREIRE, L. A. Avanços na agricultura de precisão no Brasil no período de 1999-2001. Piracicaba: Potafos, 2002. p. 58-65. 
ROBERTSON, G. P. GS ${ }^{+}$: geostatistics for the environmental sciences: version 5.1 for windows. [S.1.]: Gamma Design Software, 1998. 152 p.

SALVIANO, A. A. C.; VIEIRA, S. R.; SPAROVEK, G. Variabilidade espacial de atributos de solo e de Crotalaria juncea $(L)$ em área severamente erodida. Revista Brasileira de Ciência do Solo, Viçosa, v. 22, n. 1, p. 115-122, 1998.

SANCHEZ, R. B.; MARQUES JÚNIOR, J.; PEREIRA, G. T.; SOUZA, Z. M. Variabilidade espacial de propriedades de latossolo e da produção de café em diferentes superfícies geomórficas. Revista Brasileira de Engenharia Agrícola e Ambiental, [S.1.], v. 9, n. 4, p. 489-495, 2005.

SCHLOTZHAVER, S. D.; LITTELL, R. C. SAS: system for elementary statistical analysis. 2. ed. Cory: SAS, 1997. $905 \mathrm{p}$.

SILVA, V. R.; REICHERT, J. M.; STORCK, L.; FEIJÓ, S. Variabilidade espacial das características químicas do solo e produtividade de milho em um Argissolo Vermelho-Amarelo distrófico arênico. Revista Brasileira de Ciência do Solo, Viçosa, v. 27, n. 6, p. 1013-1020, 2003.

SIQUEIRA, G. M. Variabilidade de atributos físicos do solo determinados por métodos diversos. 2006. $163 \mathrm{f}$. Dissertação (Mestrado em Agricultura Tropical e Subtropical) - Universidade Estadual de Campinas, Campinas, 2006.

VIEIRA, S. R. Geoestatística em estudos de variabilidade espacial do solo. In: NOVAIS, R. F.; ALVAREZ, V. H.; SCHAEFER, C. E. G. R. (Eds.). Tópicos em ciência do solo. Viçosa: Sociedade Brasileira de Ciência do Solo, 2000. v. 1, p. 1-53.

WARRICK, A. W.; NIELSEN, D. R. Spatial variability of soil physical properties in the field. In: HILLEL, D. (Ed.). Applications of soil physics. New York: Academic, 1980. cap. 2, p. 319-344. 